\title{
xTAG Luminex multiplex assay for rapid screening of verocytotoxin-producing Escherichia coli strains
}

\author{
Mihaela Oprea ${ }^{1,2^{*}}$, Codruța-Romanița Usein ${ }^{2,3}$ \\ 1. The Research Institute of the University of Bucharest, Bucharest, Romania \\ 2. Molecular Epidemiology Laboratory, Cantacuzino National Institute of Medical - \\ Military Research - Development, Bucharest, Romania \\ 3. Carol Davila University of Medicine and Pharmacy, Bucharest, Romania
}

\begin{abstract}
The O26 verocytotoxin-producing Escherichia coli (VTEC)-associated outbreak of hemolytic uremic syndrome (HUS) cases in Romania during 2016 showed the need to improve the current methodology of non-O157 VTEC detection and surveillance. An in-house assay based on xTAG Luminex technology was optimized to identify seven of the most relevant diarrheagenic E.coli serogroups (O-specific wzx genes), two convenient VTEC virulence markers (eaeA and ehxA genes), and a species-specific control gene (uidA). Twenty-nine strains previously characterized in terms of serogroup and virulence genes were tested with the optimized protocol and the results were as expected. The ratio of sample signal to background varied from 66.7 (ehxA) to 7.6 (uidA) for positive samples, with a cut-off of 3. Sensitivity varied depending on the target to be amplified from approximately $10^{2}$ genomic copies to approximately $10^{4}$ genomic copies per reaction, respectively. The current approach seems an affordable alternative to commercially available assays that can be further exploited to improve existing autochthonous strategies to prevent future VTEC outbreaks.
\end{abstract}

Keywords: VTEC, bead-suspension, xTAG Luminex, rapid screening

Received: 29th January 2018; Accepted: $5^{\text {th }}$ April 2018; Published: $7^{\text {th }}$ April 2018

\section{Introduction}

Verocytotoxin-producing Escherichia coli (VTEC) are a major group of enteric pathogenic E. coli represented by zoonotic pathogens with a great propensity to cause infections with systemic complications and to generate food borne outbreaks. Their key virulence strategy is based on the capacity to produce verocytotoxins which are essential bacteriophage-encoded virulence factors, but additional mechanisms involving determinants carried on pathogenicity islands, plasmids, transposons, and phages are also responsible for pathogenesis of VTEC (1). Most VTEC strains isolated from severe cases of human infections possess the locus of enterocyte effacement (LEE), a pathogenicity island also present in the strains assigned to the enteropathogenic E. coli (EPEC) group. LEE consists

\footnotetext{
*Corresponding author: Mihaela Oprea, Cantacuzino NIMMRD, Bucuresti, Romania. E-mail: moprea@cantacuzino.ro
} 
of genes which induce the attaching and effacing lesions in the intestinal mucosa. One of them, the intimin-coding gene eae has been used as a convenient marker for LEE-positive VTEC strains. The presence of large and conserved plasmids which encode accessory virulence factors among which enterohemolysin toxin encoded by ehxA gene is common for clinically relevant strains $(2-4)$.

There are hundreds of serotypes of VTEC identified to date, but only some of them have been reported to have a high significance in human disease worldwide (5 - 8). In Europe, $E$. coli serogroups O157, O26, and $\mathrm{O} 103$ dominate human VTEC infections (http://atlas.ecdc.europa.eu/public/index.aspx). In Romania, the VTECO26 serogroup was the cause of anoutbreak of hemolytic uremic syndrome (HUS) among small children in 2016, making it the first major event linked to such pathogens $(9-10)$.

The method used for the detection of enteric pathogenic E. coli in Romanian clinical microbiology laboratories, whether public or private, is still conventional serotyping performed in a slide agglutination format with commercially available $\mathrm{O}$ antisera against EPEC and VTEC serogroups. As this procedure can be expensive and time-consuming, the strains are usually agglutinated only with polyvalent antisera and VTEC detection is focused exclusively on O157 serogroup. As a rule, the positive strains recovered from severe cases of infection are referred to the national reference laboratory for confirmation. Prior to 2016, this was the sole autochthonous laboratory that used both culture and non-culture based assays for the detection of human pathogenic $E$. coli strains in general and of VTEC in particular. The HUS outbreak challenged clinicians, microbiologists, and epidemiologists and put pressure on the public health system to improve VTEC preparedness. Consequently, new strategies for the rapid screening of a large number of $E$. coli strains in order to dis- tinguish pathogenic from non-pathogenic strains are needed.

Luminex $\quad x T A G \AA$ technology is a high-throughput, rapid, labor-saving multiplex method offering both high sensitivity and specificity. Using this technology, up to 150 different targets can be detected in a single reaction. Also, it is a flexible technology, as additional targets may be incorporated in the assay, as needed (11). Considering the commercially available kits, the local needs and the limited financial resources, this pilot study explored the use of an in-house approach based on xTAG Luminex technology for the identification of seven of the most relevant diarrheagenic Escherichia coli serogroups (targeted through wzx genes encoding the O-antigen flippase enzyme), two convenient VTEC virulence markers (eaeA and $e h x A$ genes), and $E$. coli-specific beta-glucuronidase encoding gene (uidA).

\section{Material and Methods}

\section{Bacterial strains}

The 29 bacterial strains used in this study originated from the bacterial collection of the Molecular Epidemiology Laboratory of Cantacuzino National Institute of Medical-Military Research - Development (CNIMMRD), Bucharest, Romania (Table 1). The E. coli panel included autochthonous strains isolated and identified during routine surveillance by the national reference laboratory (NRL) of CNIMMRD, as well as international E. coli strains with confirmed serogroups. The latter were distributed by the WHO Collaborating Centre for Reference and Research on Escherichia and Klebsiella from Statens Serum Institute (Denmark) to all the NRLs, including the NRL of CNIMMRD, which participated in the rounds of external quality assessment (EQA) on VTEC typing organized annually by the European Center for Disease Pre- 
vention and Control (ECDC). All strains were stored at $-70^{\circ} \mathrm{C}$ until DNA extraction.

\section{DNA extraction and quantification}

DNA extraction was performed with PureLink $^{\mathrm{TM}}$ Genomic DNA Mini Kit (Invitrogen,
Carlsbad, CA, USA), according to the manufacturer's instructions. The concentrations of purified DNA were measured with Qubit 2.0 Fluorometer (Invitrogen, Eugene, Oregon, USA), using Qubit dsDNA HS Assay Kit (Invitrogen, Eugene, Oregon, USA). All the samples were diluted to

Table 1. Bacterial strains used in this study, their serogroup and target gene content

\begin{tabular}{|c|c|c|c|c|c|}
\hline & Strain & Serogroup & eaeA & $e h x A$ & uidA \\
\hline 1 & Escherichia coli $3096^{*}$ & $\mathrm{O} 26$ & + & + & + \\
\hline 2 & Escherichia coli $3099^{*}$ & $\mathrm{O} 26$ & + & + & + \\
\hline 3 & Escherichia coli $193151^{*}$ & $\mathrm{O} 26$ & + & + & + \\
\hline 4 & Escherichia coli 3868 & $\mathrm{O} 26$ & + & + & + \\
\hline 5 & Escherichia coli 12107 & $\mathrm{O} 45$ & - & - & + \\
\hline 6 & Escherichia coli 8789 & $\mathrm{O} 103$ & + & + & + \\
\hline 7 & Escherichia coli 115 & $\mathrm{O} 103$ & + & + & + \\
\hline 8 & Escherichia coli 2206 & $\mathrm{O} 103$ & + & + & + \\
\hline 9 & Escherichia coli 9842 & $\mathrm{O} 111$ & + & - & + \\
\hline 10 & Escherichia coli 3501 & O111 & + & + & + \\
\hline 11 & Escherichia coli 2393 & O111 & + & + & + \\
\hline 12 & Escherichia coli 3390 & $\mathrm{O} 121$ & + & + & + \\
\hline 13 & Escherichia coli 2771 & $\mathrm{O} 121$ & + & + & + \\
\hline 14 & Escherichia coli 8263 & $\mathrm{O} 145$ & + & - & + \\
\hline 15 & Escherichia coli 179 & $\mathrm{O} 145$ & + & - & + \\
\hline 16 & Escherichia coli EDL933 & $\mathrm{O} 157$ & + & + & + \\
\hline 17 & Escherichia coli $213666^{*}$ & $\mathrm{O} 157$ & + & + & + \\
\hline 18 & Escherichia coli 5017 & $\mathrm{O} 157$ & + & + & + \\
\hline 19 & Escherichia coli 4062 & $\mathrm{O} 157$ & + & + & + \\
\hline 20 & Escherichia coli 117 & $\mathrm{O} 146$ & - & + & + \\
\hline 21 & Escherichia coli 172 & O91 & - & + & + \\
\hline 22 & Escherichia coli 181 & $\mathrm{O} 171$ & - & - & + \\
\hline 23 & Escherichia coli 3467 & $\mathrm{O} 104$ & - & - & + \\
\hline 24 & Escherichia coli 8145 & O166 & - & - & + \\
\hline 25 & Escherichia coli 356 & $\mathrm{O} 139$ & - & + & + \\
\hline 26 & Escherichia coli $262 *$ & unknown & - & - & + \\
\hline 27 & Escherichia coli $211986^{*}$ & unknown & - & - & + \\
\hline 28 & Campylobacter jejuni $223251 *$ & NA & - & - & - \\
\hline 29 & Staphylococcus aureus 92* & NA & - & - & - \\
\hline
\end{tabular}

* Autochthonous bacterial strains 
$10^{6}$ genomic copies/ $\mu 1$ and serial dilutions in water were performed until 1 genomic copy/ $\mu 1$.

\section{Primers for targets detection}

PrimerPlex vers. 2 software was used to design the 10 pairs of specific primers for the 10 targets (Table 2), following the instructions from Luminex (11). The xTAG assays require modified specific primers. The forward specific primers were modified by a unique 24-base oligonucleotide "TAG" sequence at the 5' terminus that is used to connect the amplicon to a specific MagPlex-xTAG microsphere (Luminex Corporation, Toronto, ON, Canada). The MagPlex-xTAG microspheres are 6.5-micron superparamagnetic beads where each bead region is covalently pre-coupled with a different 24-base oligonucleotide "anti-TAG" sequence (complementary to the "TAG"sequence). A 12-carbon spacer was incorporated between the "TAG" sequence and primer. All reverse primers were biotinylated at the $5^{\prime}$ terminus. The primer sequences, their target genes, the size of the resulting amplicons and the corresponding bead region are shown in Table 2.

\section{Multiplex PCR optimization}

Seven $E$. coli strains were used for optimization of multiplex PCR (i.e. E. coli 3096, E. coli 12107, E. coli 8789, E. coli 9842, E. coli 3390 , E. coli 8263, E. coli EDL933) selected tocover all the targets. First, the specificity of primers was confirmed in simplex PCR reactions. The multiplex PCR was influenced by many factors such as annealing time, annealing temperature, and primer concentrations. These conditions were optimized in order to assure specific amplification and sensitive detection of the targets. Finally, the multiplex reaction was set as follows: $12.5 \mu 1$ GoTaq HotStart MasterMix (Promega, Madison, WI, USA), $0.6 \mu \mathrm{M}$ uidA and O26wzx primers, $0.2 \mu \mathrm{M}$ ehxA primers, $0.1 \mu \mathrm{M}$ the other primers, and $1 \mu \mathrm{DNA}$, in a total volume of 25 $\mu 1$. Two negative controls (water) were included in each set up. To determine the sensitivity of the multiplex reaction, multiple reactions with different DNA concentrations (1-10 ${ }^{6}$ genomic copies/reaction) were performed. PCR reactions were performed with the following parameters: initial denaturation $95^{\circ} \mathrm{C}-2 \mathrm{~min}, 35$ cycles with denaturation at $94^{\circ}-30 \mathrm{sec}$, annealing at $50^{\circ} \mathrm{C}-$ $90 \mathrm{sec}$, extension at $72^{\circ} \mathrm{C}-30 \mathrm{~min}$ and final extension at $72^{\circ} \mathrm{C}-7 \mathrm{~min}$. The PCR products were then subjected to hybridization on microspheres, no more than $48 \mathrm{~h}$ later.

\section{Hybridization on MagPlex-xTAG beads}

The assay was conducted according to Luminex (11). Shortly, the hybridization mixture was set in a 96-well low-profile plate and consisted for each sample of: 2500 MagPlex-xTAG microspheres of each set, $1 \mu 1$ PCR product and $70 \mu \mathrm{l}$ Streptavidin, R-Phycoerythrin Conjugate (SAPE) (Invitrogen, Carlsbad, CA, USA) to 10 $\mu \mathrm{g} / \mathrm{mL}$, as indicator, in a final volume of $95 \mu 1$. For PCR negative controls, 2 wells for each control were assigned, leading to 4 wells with negative controls. SAPE was supplemented with BSA, to $0.1 \%$ in final volume. Hybridization was performed in a thermocycler (Veriti 96-Well Thermal Cycler, Applied Biosystems). Different hybridization parameters were tested, the final tests being performed at $40^{\circ} \mathrm{C}, 30$ minutes.

\section{Luminex 200 analyses}

Immediately after the hybridization reaction, the products were analyzed on the Luminex 200 analyzer (Luminex Corporation, Toronto, ON, Canada) according to the system manual, using xPONENT vers. 3.1 software (Luminex Corporation, Toronto, ON, Canada). The sample size was set to $70 \mu 1$, with a minimum of 100 beads per target to be analyzed. Reading was performed at $40^{\circ} \mathrm{C}$, with $90 \mathrm{~s}$ timeout, and DD (Doublet 
Table 2. Primers used in this study and corresponding MagPlex - xTAG bead region

\begin{tabular}{|c|c|c|c|c|}
\hline $\begin{array}{l}\text { Gene } \\
\text { target }\end{array}$ & Primer & Primer sequence 5'-3' & $\begin{array}{l}\text { Amplicon } \\
\text { size }\end{array}$ & $\begin{array}{c}\text { Corresponding } \\
\text { MagPlex-xTAG } \\
\text { bead region }\end{array}$ \\
\hline \multirow[t]{2}{*}{$\mathrm{O} 26$} & O26wzxF & $\begin{array}{c}\text { CAAATACATAATCTTACATTCACT }^{1)} \text {-C12)_-GCTA- } \\
\text { AAATTCAATGGGCGGAAA }\end{array}$ & \multirow[t]{2}{*}{$131 \mathrm{bp}$} & \multirow[t]{2}{*}{ A13 } \\
\hline & O26wzxR & biotin - GCAACAGGCGAAGCAAGA & & \\
\hline \multirow[t]{2}{*}{$\mathrm{O} 45$} & O45wzxF & $\begin{array}{c}\frac{\text { CATAATCAATTTCAACTTTCTACT-C12-GAGC- }}{\text { CGAGATGGTAATTCCTAA }} \\
\end{array}$ & \multirow[t]{2}{*}{$110 \mathrm{bp}$} & \multirow[t]{2}{*}{ A12 } \\
\hline & O45wzxR & biotin -GCACAACGCAACGAAAGTC & & \\
\hline \multirow[t]{2}{*}{ O103 } & O103wzxF & $\begin{array}{c}\text { AATTTCTTCTCTTTCTTTCACAAT-C12-AGGCT- } \\
\text { TATCTGGCTGTTCTTAC } \\
\end{array}$ & \multirow[t]{2}{*}{$127 \mathrm{bp}$} & \multirow[t]{2}{*}{ A14 } \\
\hline & O103wzxR & biotin - GCGAGCGGTACAACAATACA & & \\
\hline \multirow[t]{2}{*}{ O111 } & O111wzxF & $\begin{array}{c}\text { TACTTCTTTACTACAATTTACAAC-C12-TTCAAT- } \\
\text { TAACTGGTGGCGTCTC } \\
\end{array}$ & \multirow[t]{2}{*}{$102 \mathrm{bp}$} & \multirow[t]{2}{*}{ A15 } \\
\hline & O111wzxR & biotin - AGTTGAAGGCAAGGGACATAAG & & \\
\hline \multirow[t]{2}{*}{ O121 } & O121wzxF & $\begin{array}{c}\text { CAAACAAACATTCAAATATCAATC-C12-GACAAT- } \\
\text { GACAGTGCTGGACTA }\end{array}$ & \multirow[t]{2}{*}{$113 \mathrm{bp}$} & \multirow[t]{2}{*}{ A22 } \\
\hline & O121wzxR & biotin - CCGAAATGATGGGTGCTAAGA & & \\
\hline \multirow[t]{2}{*}{ O145 } & O145wzxF & $\begin{array}{l}\frac{\text { TCAAACTCTCAATTCTTACTTAAT-C12-AAGGTG- }}{\text { TATGGTGTTGGTTAGC }} \\
\end{array}$ & \multirow[t]{2}{*}{$121 \mathrm{bp}$} & \multirow[t]{2}{*}{ A 21} \\
\hline & O145wzxR & biotin - AGCCACTCCAAGCACACT & & \\
\hline \multirow[t]{2}{*}{ O157 } & O157wzxF & $\begin{array}{l}\text { CTTTCTCATACTTTCAACTAATTT-C12-CATGCAC- } \\
\text { GCAATGATACTCAAT }\end{array}$ & \multirow[t]{2}{*}{$117 \mathrm{bp}$} & \multirow[t]{2}{*}{$\mathrm{A} 20$} \\
\hline & O157wzxR & biotin - AACGACTTCACTACCGAACAC & & \\
\hline \multirow{2}{*}{ eaeA } & eaeAF & $\begin{array}{l}\frac{\text { ACACTTATCTTTCAATTCAATTAC-C12-ATG- }}{\text { GAACGGCAGAGGTTAATC }} \\
\text { GACATA }\end{array}$ & \multirow[t]{2}{*}{$149 \mathrm{bp}$} & \multirow[t]{2}{*}{ A18 } \\
\hline & eaeAR & biotin -CGTAAAGCGGGAGTCAATGT & & \\
\hline \multirow[t]{2}{*}{$e h x A$} & ehxAF & $\begin{array}{l}\text { CTTTCTTAATACATTACAACATAC-C12-AGAATG- } \\
\text { GATATGACGCAAGACA }\end{array}$ & \multirow[t]{2}{*}{$139 \mathrm{bp}$} & \multirow[t]{2}{*}{ A 25} \\
\hline & ehxAR & biotin - GCAAGTTCACCGATCTTCTCA & & \\
\hline \multirow{2}{*}{ uidA } & uidAF & $\frac{\text { ATACTTTACAAACAAATAACACAC-C12-AATG- }}{\text { GTGATGTCAGCGTTGAA }}$ & \multirow[t]{2}{*}{$105 \mathrm{bp}$} & \multirow[t]{2}{*}{ A19 } \\
\hline & uidAR & biotin - TTGCCAGAGGTGCGGATT & & \\
\hline
\end{tabular}

${ }^{1)}$ TAG sequences - underline

2)12 Carbon spacer - HEG (hexaethylene glycol)

Discriminator) gate $7500-13500$ with the High PMT (PhotoMultiplierTube) selected to increase the reporter gain level. The results were reported as median fluorescence intensity (MFI) of each microsphere region. From the MFI, signal to background ratios were calculated (signal_MFI/
background_MFI), where background MFI value is obtained from the 4 negative control MFIs using the formula: Background MFI = MFIMe$\mathrm{an}_{\mathrm{NC}}+1.645\left(\mathrm{SD}_{\mathrm{NC}}\right)$. Samples were considered to be positive when signal to background ratio was greater than 3.0. 


\section{The evaluation of sensitivity}

To determine the sensitivities of the multiplex assay, serial dilutions (1-106 genomic copies/ $\mu 1$ ) of the 7 bacterial DNA samples used for optimization were tested. The sensitivity results were confirmed in three different experiments.

\section{The evaluation of specificity}

To assess the specificity of the assay, all 29 bacterial strains considered for this study were tested. Approximately $5 \times 10^{6}$ genomic DNA copies were introduced in each reaction.

\section{Results}

\section{Specificity of the multiplex reaction}

The antigenic and virulence characteristics of the 29 bacterial strains tested with the optimized protocol were as expected. The ratio of sample signal to background varied from 66.7 $(e h x A)$ to 7.6 (uidA) for positive samples. Table 3 presents the signal to background ratio values of the positive targets. When multiple samples were positive for the same target, the ratio values are indicated as the mean \pm standard error of the mean. When the samples were negative, the signal to background ratio was below 3.0.

\section{Sensitivity of the multiplex reaction}

The sensitivity varied depending on the target to be amplified: $10^{2}$ genomic copies (about $5 \mathrm{pg}$. of DNA) for O45wzx, O111wzx, O145wzx and $\mathrm{O} 157 w z x, 10^{3}$ genomic copies for $\mathrm{O} 26 w z x$, O103wzx, O121wzx, ehxA, eaeA, and $10^{4}$ genomic copies for uidA target. The sensitivity was calculated plotting the median of positive sample to background ratio of each target to a cut-off of 3.0 (Figure 1).

\section{Discussion}

Although there is a wide variety of laboratory assays available for VTEC identification, the problems related to the diagnostic of VTEC infections are still challenging $(5,12-14)$. The choice of the methods utilized is not easy to make, which is the reason why there is a lack of standardization in the approach of identification of human VTEC strains, especially for non-O157 VTEC.

In the ECDC zoonosis reports published annually, Romania has a very low reported incidence of VTEC (15). Although this incidence is most likely to be real, the reported epidemiology might be undoubtedly influenced by the efficiency of the laboratory practice. The outbreak of VTEC infections that occurred in 2016 disquieted the public health system revealing the inability to quickly recognize and respond to such diseases. In the attempt to optimize the diagnosis for VTEC in Romania, we considered the advantages of using the Luminex xTAG ${ }^{\circledR}$ platform as the equipment was available but not previously used for diagnostic assays for infectious diseases.We were aware of the existence of the commercially available kits that screened for VTEC using Luminex platform. Luminex xTAG ${ }^{\circledR}$ Gastrointestinal Pathogen Panel (xTAG® GPP) (16) was one of them, which was able to identify major gastrointestinal pathogens, VTEC included, and to discriminate members of VTECO157 serogroup. Another one, more recently introduced, xMAP ${ }^{\circledR}$ Molecular STEC Serotyping Assay, screened not only for $E$. coli strains of serogroup O157, but also of other non-O157 serogroups, frequently associated with VTEC, along with the

Table 3. Sample signal to background ratio for positive samples

\begin{tabular}{lcccccccccc}
\hline \multirow{2}{*}{ Target } & O26 & O45 & O103 & O111 & O121 & O145 & O157 & \multirow{2}{*}{ eaeA } & ehxA & uidA \\
\hline Sample signal/ & $w z x$ & $w z x$ & $w z x$ & $w z x$ & $w z x$ & $w z x$ & $w z x$ & & & \\
background & 11.2 & \multirow{2}{*}{48.7} & 12.8 & 52.1 & 54.4 & 53.2 & 42 & 22.1 & 54.0 & 10.6 \\
\hline
\end{tabular}




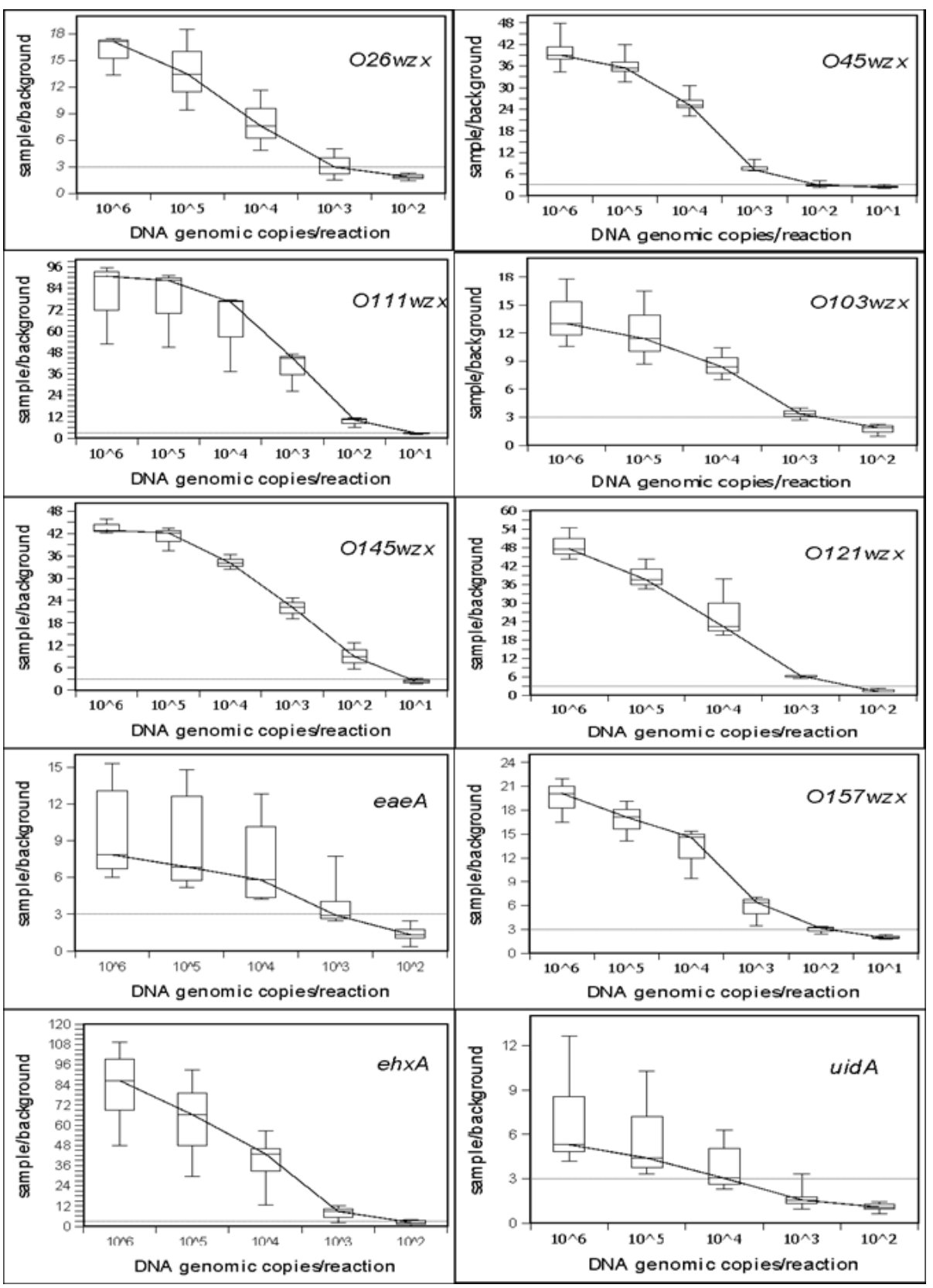

Figure 1. Sensitivity of detection for each target from multiplex assay. Cut-off for sample signal to background ratio was set to 3.0

eae and $\operatorname{agg} R$ (transcriptional regulator) genes. However, to our knowledge, there was no laboratory-based evaluation of this kit, in terms of specificity and sensitivity, available before our report about this in-house assay. Thus, based on the results presented here, our XTAG assay has a great advantage over the already available commercial kits at least in terms of flexibility, be- 
ing able to be adjusted (e.g. expanded to include more targets), as needed, in order to increase its diagnostic capability. Moreover, as no target specific labels are added to the stock xTAG beads, these could be used in other XTAG assays as well, thus diminishing the cost of the assays.

Convinced by the results of previously published studies about the added value of beadbased multiplexed molecular testing (17 - 20) we chose to develop an in-house protocol for the rapid screening of the $\mathrm{O}$ serogroups known to most frequently include VTEC strains with epidemiological relevance. Seven VTEC relevant serogroups were targeted in the presented assay. Considering the heterogeneity of these $E$. coli serogroups, a predictive screening for two $E$. coli virulence genes was associated in order to distinguish pathogenic from non-pathogenic strains. Additionally, the uidA gene target was included in order to provide an opportunity if necessary to skip preliminary tests for the E. coli species identification.

The number of strains included in the panel used for the validation of our in-house protocol was small which was considered a limitation to our study. Yet, these first accurate and reproducible results obtained in less than 4 hours led us to consider the assay reliable. In terms of sensitivity, the detection limit calculated to be from $10^{2}$ to $10^{4}$ genomic copies for the various gene targets was considered acceptable for our needs, as the assay was intended for testing $E$. coli strains/ cultures and not stool specimens. Whereas the validation could be further expanded on a higher number of clinical isolates in order to be sure of the assay specificity, the cost associated with the microspheres used in such a complex multiple detection could still be a limitation for the future use in routine diagnostic. Nevertheless, this drawback could be overcome when large batches of strains are tested, as may be the case in an outbreak.
Overall, the current approach seems to be an affordable alternative to commercially available assays that can be further exploited to improve existing autochthonous strategies to prevent future VTEC outbreaks.

\section{Funding}

This work was supported by The Research Institute of the University of Bucharest, Romania (ICUB Fellowships for Young Researchers awarded to M. O., November 2016) and by Cantacuzino National Institute of Medical-Military Research - Development, Bucharest, Romania.

\section{Conflict of interest disclosure}

None of the authors has any conflict of interest to declare.

\section{References}

1. Etcheverría AI, Padola NL. Shiga toxin-producing Escherichia coli: factors involved in virulence and cattle colonization. Virulence. 2013;4:366-72. DOI: 10.4161/viru.24642

2. Croxen MA, Law RJ, Scholz R, Keeney KM, Wlodarska M, Finlay BB. Recent advances in understanding enteric pathogenic Escherichia coli. Clin Microbiol Rev. 2013;26(4):822-80. DOI: 10.1128/CMR.0002213

3. Kaper JB, Nataro JP, Mobley HL. Pathogenic Escherichia coli. Nat Rev Microbiol. 2004;2(2):123-40. DOI: 10.1038/nrmicro818

4. Scheutz F, Strockbine NA. Escherichia. In: Garrity GM, Brenner DJ, Krieg NR, Staley JT, editors. Bergey's manual of systematic bacteriology. New York: Springer; 2005. P. 607-624.

5. Fratamico PM, DebRoy C, Liu Y, Needleman DS, Baranzoni GM, Feng P. Advances in molecular serotyping and subtyping of Escherichia coli. Front Microbiol. 2016;7:644. DOI: 10.3389/fmicb.2016.00644

6. Iguchi A, Iyoda S, Kikuchi T, Ogura Y, Katsura K, Ohnishi $\mathrm{M}$ et al. A complete view of the genetic diversity of the Escherichia coli O-antigen biosynthesis gene cluster. DNA Res 2015;22(1):101-7. DOI: 10.1093/ dnares/dsu043

7. Johnson KE, Thorpe CM, Sears CL. The emerging clinical importance of non-O157 Shiga toxin-producing Escherichia coli. Clin Infect Dis 2006;43:1587-95. DOI: $10.1086 / 509573$

8. Muniesa M, Hammerl JA, Hertwig S, Appel B, Brüssow 
H. Shiga toxin-producing Escherichia coli O104:H4: a new challenge for microbiology. Appl Environ Microbiol 2012;78(12):4065-73. DOI: 10.1128/AEM.0021712

9. Peron E, Zaharia A, Zota LC, Severi E, Mårdh O, Usein $\mathrm{C}$, et al. Early findings in outbreak of haemolytic uraemic syndrome among young children caused by Shiga toxin-producing Escherichia coli, Romania, January to February 2016. Euro Surveill 2016;21(11):30170. DOI: 10.2807/1560-7917.ES.2016.21.11.30170

10. Usein CR, Ciontea AS, Militaru CM, Condei M, Dinu S, Oprea M, et al. Molecular characterisation of human Shiga toxin-producing Escherichia coli O26 strains: results of an outbreak investigation, Romania, February to August 2016. Euro Surveill 2017;22(47):17-00148. DOI: $10.2807 / 1560-7917 . E S .2017 .22 .47 .17-00148$

11. Luminex $x M A P \AA$ Cookbook. A collection of methods and protocols for developing multiplex assays with xMAP Technology. 2rd Edition. Available at http://info. luminexcorp.com/en-us/download-the-xmap-cookbook Accessed November 14, 2016.

12. DebRoy C, Roberts E, Valadez AM, Dudley EG, Cutter CN. Detection of Shiga toxin-producing Escherichia coli O26, O45, O103, O111, O113, O121, O145, and O157 serogroups by multiplex polymerase chain reaction of the wzx gene of the O-antigen gene cluster. Foodborne Pathog Dis 2011;8(5):651-2. DOI: 10.1089/ fpd.2010.0769

13. Leszczyńska B, Ziółkowska H, Podsiadły E, Szych J, Rastawicki W, Demkow U, et al. Diagnostic value of serological tests against verotoxigenic Escherichia coli in hemolytic uremic syndrome in children. Adv Clin Exp Med. 2015;24(6):1031-6. DOI: 10.17219/ acem/41931

14. Rice T, Quinn N, Sleator RD, Lucey B. Changing diagnostic methods and increased detection of verotoxigenic Escherichia coli, Ireland. Emerg Infect Dis. 2016;22(9):1656-7. DOI: 10.3201/eid2209.160477

15. European Centre for Disease Prevention and Control. Annual Epidemiological Report 2016 - Shigatoxin/ verocytotoxin-producing Escherichia coli infection. Available at http://ecdc.europa.eu/en/healthtopics/stec/ Pages/Annual-epidemiological-report-2016.aspx Accessed December 20, 2017.

16. Wessels E, Rusman LG, van Bussel MJ, Claas EC. Added value of multiplex Luminex Gastrointestinal Pathogen Panel (xTAG ${ }^{\circledR}$ GPP) testing in the diagnosis of infectious gastroenteritis. Clin Microbiol Infect 2014;20(3):O182-O187. DOI: 10.1111/14690691.12364

17. Carter JM1 Lin A, Clotilde L, Lesho M. Rapid, multiplexed characterization of Shiga toxin-producing Escherichia coli (STEC) Isolates Using Suspension Array Technology. Front Microbiol 2016;7:439.

18. Jiang L, Ren H, Zhou H, Qin T, Chen Y. Simultaneous detection of nine key bacterial respiratory pathogens using Luminex xTAG(®) technology. Int J Environ Res Public Health 2017;14(3):E223. DOI: 10.3390/ ijerph14030223

19. Lin A, Nguyen L, Lee T, Clotilde LM, Kase JA, Son I, Carter JM, Lauzon CR. Rapid O serogroup identification of the ten most clinically relevant STECs by Luminex microbead-based suspension array. J Microbiol Methods 2011;87(1):105-10. DOI: 10.1016/j.mimet.2011.07.019

20. Clotilde LM, Bernard C 4th, Hartman GL, Lau DK, Carter JM. Microbead-based immunoassay for simultaneous detection of Shiga toxins and isolation of Escherichia coli O157 in foods. J Food Prot 2011;74(3):3739. DOI: 10.4315/0362-028X.JFP-10-344 